Supporting Information

\title{
Coiled optical nanofiber for optofluidic absorbance detection
}

\author{
Hongyan Mei, Jing Pan, Zhang Zhang, Lei Zhang* and Limin Tong* \\ State Key Laboratory of Modern Optical Instrumentation, College of Optical Science and \\ Engineering, Zhejiang University, Hangzhou 310027, China \\ Correspondence to: zhang_lei@zju.edu.cn; phytong@zju.edu.cn
}

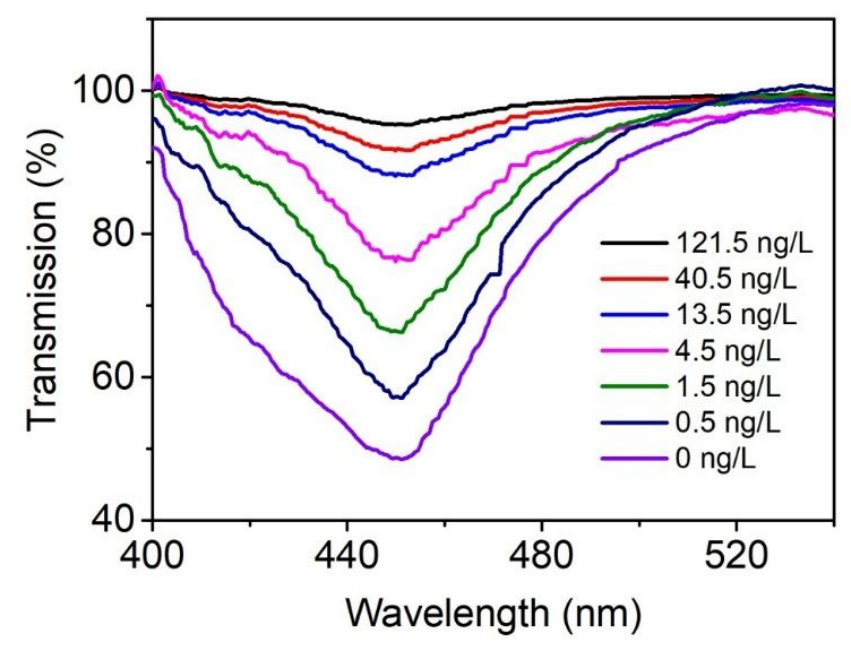

Figure S1 Transmission spectra of CAP standards ( 0 to $121.5 \mathrm{ng} / \mathrm{L}$ ) recorded by the nanofiber sensor. 